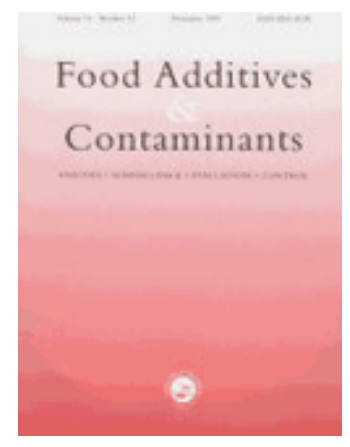

\title{
A toxicokinetic model for the carry-over of dioxins and PCBs from feed and soil to eggs
}

\begin{tabular}{|r|l|}
\hline Journal: & Food Additives and Contaminants \\
\hline Manuscript ID: & TFAC-2005-177.R1 \\
\hline Manuscript Type: & Original Research Paper \\
\hline Author: & O2-Dec-2005 \\
\hline Complete List of Authors: & $\begin{array}{l}\text { Hoogenboom, Ron; RIKILT-Institute of Food Safety, Safety and } \\
\text { Health } \\
\text { van Eijkeren, Jan; RIVM } \\
\text { Zeilmaker, Marco; RIVM } \\
\text { Kan, Kees; Animal Science Group of Wageningen UR, Animal } \\
\text { Husbandry } \\
\text { Traag, Wim; RIKILT-Institute of Food Safety }\end{array}$ \\
\hline Methods/Techniques: & Regulations, Toxicology - pharmokinetics \\
\hline Additives/Contaminants: & Dioxins, PCBs \\
\hline Food Types: & Animal feed, Eggs \\
\hline & \\
\hline
\end{tabular}

\section{SCHOLARONE ${ }^{\text {M }}$ \\ Manuscripts}


6 A toxicokinetic model for the carry-over of dioxins and PCBs

\section{7 from feed and soil to eggs}

8

9

10

11

12

13 J.C.H. van Eijkeren ${ }^{1 *}$, M.J. Zeilmaker ${ }^{1}$, C.A. $\mathrm{Kan}^{2}$, W.A. Traag ${ }^{3}$ \& L.A.P. Hoogenboom ${ }^{3}$ 14 15

16

171 RIVM National Institute of Public Health and the Environment, P.O.Box 1, 3720 BA, Bilthoven, The $18 \quad$ Netherlands

192 Animal Sciences Group of Wageningen UR, Division Nutrition and Food, P.O. Box 65, 8200 AB $20 \quad$ Lelystad, The Netherlands

213 RIKILT-Institute of Food Safety, Bornsesteeg 45, 6708PD Wageningen, The Netherlands

22

23

24

25

$26 *$ To whom correspondence should be addressed. e-mail:jan.van.eijkeren@rivm.nl 


\section{Abstract}

28 A mathematical model for the kinetics of carry-over of dioxins and dioxin-like PCBs from 29 feed mixed with contaminated oil to eggs has been developed. This model incorporates 30 uptake of the compounds over the gut wall and their subsequent transport by blood, 31 distribution over the body, hepatic metabolism and excretion through egg yolk fat. The 32 model is analysed with respect to the possibility of identifying yet unknown model 33 parameters by fitting these to the experimental data. The model was fitted to the 34 experimental data on the carry-over from feed to eggs. The calibrated model was applied to 35 calculate the steady-state concentrations in eggs which were compared to European 36 Maximum Residue Levels for dioxins in feed and eggs, showing that these limits do not 37 match. The feed limit of $0.75 \mathrm{ng} \mathrm{TEQ} / \mathrm{kg}$ should be reduced to about $0.17 \mathrm{ng} \mathrm{TEQ} / \mathrm{kg}$ in 38 order to guarantee egg levels below the residue limit of $3 \mathrm{pg} \mathrm{TEQ} / \mathrm{g}$ fat. Experimental 39 results of carry-over from contaminated soil were used to estimate the absorption of 40 dioxin-like compounds from soils as compared to the absorption from feed, resulting in a 41 value around 40 to $60 \%$ absorption from soil as compared to around $90 \%$ absorption from 42 feed.

45 Key words:- Dioxins, PCBs, chicken, feed, soil, PBPK-model 
46

47

48

\section{Introduction}

Controlled exposure studies under laboratory conditions have been performed to examine carry-over of dioxins and dioxin-like PCBs to eggs from feed mixed with contaminated oil and from clean feed mixed with contaminated soil (Hoogenboom et al., 2005). The experiments with contaminated feed not only served the determination of steady state carry-over, but the study of the kinetics of carry-over as well. The work also aimed to developm a computer model to describe carry-over. Only a few authors have until now described a model to relate contamination levels of eggs to the contamination of ingested soil or feed. Schuler et al. (1997) presented the calculation of total TEQ contamination of eggs from ingestion of soil, based on soil contamination levels, congener specific transfer efficiencies and background feed contamination levels. Their model did not contain time as a variable and implicitly assumed steady state contamination of the eggs. However, egg contamination caused by uptake from contaminated soil or feed is a kinetic process and the apparent contamination levels may not represent steady state. Huygebaert et al. (2002) developed a kinetic model for the excretion of PCBs in egg yolks from contaminated feed. Their model assumption was that excretion is governed by the hydraulics of, and absorption in the animal intestinal tract. However, not only absorption of contaminants over the intestinal wall is an important aspect for the kinetics of egg contamination, but also the distribution of the contaminant over body tissues, its metabolism and the characteristics of excretion, i.e. laying efficiency and, for highly lipophilic contaminants like dioxins, egg lipid content.

This study concerned the modelling of transfer of dioxins and dioxin-like PCBs from contaminated feed to eggs and their accumulation in fat tissue. A kinetic model was developed that considers the uptake of the contaminant, i.e. its absorption over the intestinal wall after oral intake, the distribution of the contaminant over the body and its 
71 elimination by hepatic metabolism and excretion by egg yolk fat. Experimental data on 72 carry-over from feed to eggs provided by Hoogenboom et al. (2005) were used to calibrate 73 the model, relating residues in eggs to exposure through feed at different contamination 74 levels. Data obtained from Hoogenboom et al. (2002), obtained from a study with much 75 higher dioxin and PCB levels, served model verification. The calibrated and validated 76 model has been applied to estimate uptake from another matrix, i.e., from contaminated 77 soil added to clean feed. Moreover, the model was applied for comparing the European 78 Maximum Residue Limits for dioxin residues (expressed as TEQ values) in feed and eggs. 
79

80

81

86

\section{Materials and method}

\section{Experimental}

All data for calibrating the model were obtained from the experiment described in the accompanying paper by Hoogenboom et al. (2005). Verification data were obtained from the study by Hoogenboom et al. (2002).

\section{$\underline{\text { Modelling approach }}$}

The underlying physiologically based pharmacokinetic (PBPK-)model describing the kinetics of carry-over of contaminants from feed to eggs is depicted in figure 1 (upper panel). The corresponding compartment model in figure 1 (lower panel) can be formulated mathematically as a set of two mass balances, representing the changes in absolute amounts of contaminants in respectively the central and the fat compartment.

$$
\begin{aligned}
& \frac{d A_{c}}{d t}=F_{a b s} D-\left(q_{c}+\varepsilon y+k\right) A_{c}+q_{f} A_{f} \\
& \frac{d A_{f}}{d t}=q_{c} A_{c}-q_{f} A_{f}
\end{aligned}
$$

93 Here, the reduced parameters expressed in terms of the unknown PBPK-model parameters

$$
q_{c}=\frac{Q}{P_{c} V_{c}}, \quad q_{f}=\frac{Q}{P_{f} V_{f}}, \quad y=\frac{P_{y, f} W_{y, f}}{P_{c} V_{c}} \text { and } k=\frac{C L}{P_{c} V_{c}}
$$

95 are the rate constants of mass transfer from the central to the fat compartment and vice versa, excretion with egg yolk fat and hepatic clearance, respectively.

97 The time course of the concentration in egg yolk, expressed as $\mathrm{pg} \mathrm{TEQ} / \mathrm{g}$ yolk fat 98 and in abdominal fat (pg TEQ / $\mathrm{g}$ fat) is observed. If the latter is representative of the 99 residue levels in the fat compartment, than the models for these observations, can be derived from equation ( 1 ) to be 


$$
C_{y, f}(t)=\frac{y}{W_{y, f}} \cdot \frac{F_{a b s} D}{r}\left(1-\left(\frac{\left(\lambda_{2}+r\right) e^{\lambda_{1} t}-\left(\lambda_{1}+r\right) e^{\lambda_{2} t}}{\lambda_{2}-\lambda_{1}}\right)\right)
$$

102 for the concentration in egg yolk fat, and

$$
C_{f}(t)=\frac{1}{W_{f}} \cdot \frac{q_{c}}{q_{f}} \frac{F_{a b s} D}{r}\left(1-\left(\frac{\lambda_{2} e^{\lambda_{1} t}-\lambda_{1} e^{\lambda_{2} t}}{\lambda_{2}-\lambda_{1}}\right)\right)
$$

104 for the concentration in abdominal fat. Here, $r=\varepsilon y+k$ represents the total elimination of

105 the compounds and

106

$$
\begin{aligned}
& \lambda_{1}=-\frac{1}{2}\left(q_{c}+r+q_{f}-\sqrt{\left(q_{c}+r+q_{f}\right)^{2}-4 q_{f} r}\right) \\
& \lambda_{2}=-\frac{1}{2}\left(q_{c}+r+q_{f}+\sqrt{\left(q_{c}+r+q_{f}\right)^{2}-4 q_{f} r}\right)
\end{aligned}
$$

107 are the exponential rates of the slow, long lasting, terminal phase $\left(\lambda_{1} \approx q_{f} r /\left(q_{c}+q_{r}+r\right)\right.$ and 108 the fast, short lasting, initial phase $\lambda_{2} \approx q_{c}+q_{r}+r$.

109 Note, that the experimental data necessitate the introduction of a few additional 110 parameters with respect to the model in equation (1), such as the amount of fat in egg yolk,

111 which is known, and the weight of the fat compartment, which is unknown. As a result, the

112 model contains the four unknown kinetic parameters for transport and elimination, $q_{c}, q_{f}, k$ 113 and $y$, the unknown absorbed fraction $F_{a b s}$ and the unknown fat compartment weight, $W_{f}$.

114 Straightforward analysis shows that the inter compartment transfer rate parameters $115 q_{c}$ and $q_{f}$ can be identified unconditionally from the data. Therefore, these parameters can 116 be freely varied to fit the data. However, the elimination rate parameters $y$ and $k$, the 117 fraction absorbed and the fat compartment weight $W_{f}$ can only be determined in relation to 118 each other, i.e., the parameters

$$
c_{1}=r=\varepsilon y+k, \quad c_{2}=y \cdot F_{a b s} \text { and } c_{3}=F_{a b s} / W_{f}
$$

120 could be varied freely to fit the data, but not the parameters $y, k, F_{a b s}$, and $W_{y}$ separately.

121 Note that $c_{1}$ denotes the total elimination from the system of the amount that is absorbed 
122 over the gut wall, $c_{2}$ denotes the elimination through eggs of the amount that is ingested by

123 the hens and $c_{3}$ relates the experimntally determined abdominal fat concentrations to the

124 unknow fraction of the contaminant that absorbed.

125 Without introducing these new parameters of equation (6) into the computer model, this 126 problem can be resolved as follows. The fraction absorbed is 1 at maximum, while the 127 metabolism rate is 0 at minimum. If the model is fit to the data while the metabolism rate is

128 kept constant, $k=0$, then the maximum value for the excretion rate $y_{\max }=c_{1}$ can be 129 determined from the data, as well as the minimum fraction absorbed, $F_{a b s, \min }$, and the 130 minimum weight of the fat compartment $W_{y, m i n}$. This would represent the case that 131 elimination by metabolism is negligible compared to elimination through eggs. Once these 132 parameters are identified under this restrictive condition, then for $F_{a b s, \max }=1$, i.e., 133 representing the case that the amount ingested is totally absorbed over the gut wall, the 134 minimum value for the excretion rate is found to be the product $y_{\min }=y_{\max } . F_{a b s, \min }$ of the 135 values found for the case $k=0$. From this minimum, the maximum value for the 136 metabolism rate $k_{\max }=y_{\max }-y_{\min }$ can be found. The maximum value of the fat compartment 137 weight follows from $W_{f, \max }=W_{f, \min } / F_{a b s, \min }$.

138 It is assumed that after ovulation, the yolk is not any more contaminated during egg

139 white formation and deposition. Therefore, one should provide for a one-day delay 140 between ovulation of the yolk and laying of the corresponding egg. Besides, while in the 141 long range, the daily loss by excretion is the product of laying efficiency and excretion per 142 day, i.e. $\varepsilon y$, the amount to be found in an excreted egg is proportional to $y$ only. Moreover, 143 one should provide for the time, $\tau$, between the start of feeding contaminated feed and the 144 first yolk ovulation thereafter, which is apparent as an off-set in the time course of egg 145 contamination. This latter parameter is estimated from the data too. 


\section{Results and Discussion}

147 The underlying physiologically based pharmacokinetic (PBPK-)model describing the

148 kinetics of carry-over of contaminants from feed to eggs is depicted in figure 1. The fat

149 compartment comprises abdominal fat, subcutaneous fat, fat fraction of the skin, bone

150 marrow and intermuscular fat. This compartment is characterized by its storage capacity,

151 because of the extreme lipophilicity of the compounds, and by its relatively poor blood

152 flow that may even be further limited by intra-tissue diffusion. Therefore, equilibrium

153 between the concentration in this compartment and the concentration in blood is likely to

154 be reached much slower, typically in the order of a few days, than equilibrium between the

155 concentration in blood and other tissues.

156 The central compartment comprises all the other tissues, which will reach

157 equilibrium with blood in the order of hours rather than in the order of days. After

158 absorption, the contaminants enter the systemic blood circulation via the vena porta, i.e.,

159 into the central compartment $\left(F_{a b s} D\right)$. Biotransformation of the contaminants is assumed to

160 take place in the liver, i.e. the central compartment, which is characterized by the

161 parameter $C L$ (for clearance). Excretion by egg yolk is from the blood, i.e., from the

162 central compartment into yolk. The concentration in the growing yolks is assumed to be

163 proportional to the concentration in blood (partition coefficient $P_{y, f}$ ). A model, based on

164 the work of Donoghue and co-workers (Donoghue et al., 1996, 1997a, 1997b; Donoghue

165 and Meyers, 2000; Donoghue, 2001) that incorporated also yolk growth as determinant of

166 the concentration had to be abandoned, because it systematically under-estimated yolk

167 contamination levels during the initial phase. After ovulation, the yolk is assumed to be

168 excreted from the central system, because the lipophilic contaminants are not likely to

169 interact between system and yolk during the phase of white formation. This is in contrast 
170 with non-lipophilic drugs. Yolk fat of weight $W_{y, f}$ is produced with an efficiency of $\varepsilon$ per 171 day. Laying efficiency is determined experimentally and thus is a known model parameter.

172 Eggs in the trial weighed about 60 grams in the mean, of which according to Gilbert (1971) $17332 \%$ was assumed to be yolk weight) and $30 \%$ of yolk weight to be egg yolk fat weight, 174 amounting to $5.8 \mathrm{~g}$ of yolk fat per egg. Laying performance during the experiment was 175 about $90 \%$. Data on total TEQs, i.e., the sum TEQs of dioxins, furans, mono- and non176 ortho PCBs, as presented in the accompanying article (Hoogenboom et al. 2005), were 177 used for further calibrating the model. This consisted of fitting the two transfer rate 178 constants, $q_{c}$ and $q_{f}$, the egg yolk excretion rate $y$, the fraction absorbed $F_{a b s}$ and the fat 179 compartment weight $W_{f}$, while keeping the elimination constant $k=0$. The model was 180 fitted to both the data on egg yolk and abdominal fat levels of all the different feed 181 contamination levels simultaneously. Therefore, all the available data served model fitting 182 in only one optimization run, optimizing the log likelihood of the fitting parameter set. As 183 yolks ovulate the day before the eggs are sampled, eggs yolk levels analyzed from time $t$ 184 (day), were compared to computed levels at time $t-1$ (day). Moreover, as ovulation after 185 the start of applying contaminated feed, considered to be $t=0$, takes place at an unknown 186 time, another parameter, $\tau<1$ (day), was fitted to the data. Data fitting was performed with 187 ACSL Optimize, optimizing the log likelihood of the parameter values. Fitted values were 188 automatically converted to values for the other extreme case when $F_{a b s}=1$, meaning 189 complete absorption of the contaminants. Parameter values and ranges of values found by 190 fitting are:

$$
\begin{aligned}
& \left.q_{c}=0.17[-/ \text { day }], \quad q_{f}=0.078[-/ \text { day }], \quad \tau=0.19 \text { [day }\right] \\
& 0.043 \leq y \leq 0.055[-/ \text { day }], \quad 0 \leq k \leq 0.012[-/ \text { day }] \\
& 0.78 \leq F_{a b s} \leq 1[-] \text { and } 230 \leq W_{f} \leq 290[\mathrm{~g}]
\end{aligned}
$$


192 of which the first line shows unconditional results, while the resulting interval estimates in

193 the second and third line are mutually dependent. Taking e.g. the minimum possible value

194 for metabolism, $k=0$, the corresponding value for excretion is maximal and corresponding

195 values for absorption and fat compartment weight are minimal. The value for the mean

196 ovulation time $\tau$ for the population was 0.19 day after the start of feeding contaminated

197 feed, being about four and a half hours. Clearly, considering the values for $y$ and $k$, the

198 main route of elimination of dioxins from the body is by egg yolk fat and not metabolism.

199 The fact that the transfer parameter from the central to the fat compartment $q_{c}$ is higher

200 than $q_{f}$ implies that the distribution volume $\left(P_{f} V_{f}\right)$ of the fat compartment, i.e., its

201 physiological volume corrected for its storage capacity as compared to the same volume of

202 blood, is greater than the distribution volume $\left(P_{c} V_{c}\right)$ of the central compartment.

203 Figure 2 shows the residue data and the computed concentration-time curves for the

204 total TEQ levels in both egg yolk and abdominal fat based on the fitted parameter values.

205 Figure 3 shows similar curve fits for the total TEQ, but also the TEQ levels derived from

206 the dioxins and furans, the non-ortho and the mono-ortho PCBs. Notice the clear bi-phasic

207 time course of egg residue levels, while abdominal fat levels seem to be mono-exponential.

208 The (short-lasting) fast first phase has a half-life of about 2.5 days. The (long-lasting) slow

209 terminal phase appeared to have a terminal half-life of about 50 days. Fat storage behaves

210 like a large capacitor, with slowly reacting kinetics, while egg levels, that are proportional

211 to blood levels, reflect the small capacitance, fast reaction kinetics of blood levels. This

212 phenomenon appears not only during the period of feeding contaminated feed, but also

213 during the stage thereafter. The apparent difference in the kinetics of residues in eggs and

214 abdominal fat is also depicted in Figure 4 for the $1.85 \mathrm{ng}$ TEQ/kg feed contamination

215 group. Note that during the contamination period, abdominal fat residue levels are lower

216 then egg residue levels, and vice versa during the depletion period on clean feed. After 
217 prolonged feeding with contaminated feed, the steady state concentrations would reach 218 about the same level (lower panel).

219 Based on the model parameters found by fitting the model to the data, the 220 concentration-time courses for total TEQ, total dioxin TEQ, non-ortho PCB TEQ and 221 mono-ortho PCB TEQ were calculated for the experiment with highly contaminated feed 222 from Hoogenboom et al. (2002). Note that in this study the relative contribution of 223 dioxins,non-ortho PCBs, and mono-ortho-PCBs to the total TEQ level was quite different 224 from the gross composition of the contamination in the current experiment, being 225 respectively 31,12 and 58\%, as compared to 53, 29 and $18 \%$. In addition, the total TEQ 226 level of about $200 \mathrm{ng} \mathrm{TEQ} / \mathrm{kg}$ feed was 50 times higher than the maximum level in this 227 experiment: $3.95 \mathrm{ng} \mathrm{TEQ} / \mathrm{kg}$ feed. The result of the verification is depicted in figure 5, 228 showing an quite satisfactory fit of the data.

The model was subsequently used to compare EC Maximum Residue Limits for 230 dioxin TEQ residue levels in feed (0.75 $\mathrm{ng} \mathrm{TEQ} / \mathrm{kg}$ feed) and in eggs (3 pg TEQ/g fat). 231 From the former, the corresponding steady-state egg contamination level was calculated to 232 be $13 \mathrm{pg}$ TEQ/g fat, to be compared with the MRL value of $3 \mathrm{pg}$ TEQ/g fat. On the other 233 hand, maintaining the MRL value of $3 \mathrm{pg}$ TEQ/g fat in eggs, feed levels should not exceed $2340.17 \mathrm{ng}$ TEQ/kg feed, being about 4 times lower than the MRL value of $0.75 \mathrm{ng}$ TEQ/kg 235 feed. Based on current levels in battery eggs, this seems to be quite achievable.

236 Furthermore, the model was employed to estimate the fraction of dioxins absorbed 237 from two contaminated soils incorporated in the feed as described by Hoogenboom et al. 238 (2005). Keeping all other parameters at the value of the calibrated model, the fraction 239 absorbed was derived from the data. Absorption from the two soils appeared to be less 240 efficient than absorption from contaminated oil mixed into the feed being respectively $40 \%$ 
241 for one of the soils and 60\% for the other, as compared to about $90 \%$ (estimated range: 80 242 to $100 \%$ ) for the oil contaminated feed.

243 In the current study, indicator PCBs (PCBs 28, 53, 101, 118, 138, 153 and 180)

244 were also spiked to the oil used for preparing the feed. Figure 6 shows the data for total 245 indicator PCB levels in eggs and abdominal fat together with a curve fit. Parameters had to 246 be slightly modified, since without modification there was a clear overestimation of the 247 egg levels (Table 1). The reduced value of notably $q_{f}$ shows that the distribution volume 248 of the fat compartment for these indicator PCBs is greater than for dioxins and dioxin-like 249 PCBs, resulting in a greater storage capacity and reduction of residue levels. 250 Corresponding half-lives of the initial and terminal phase are 2.8 days and 55 days, 251 respectively, ten percent increased as compared to the values of 2.5 and 50 days for dioxins 252 and dioxin-like PCBs.

\section{Conclusions}

255 - A model was developed for the kinetics of total sum TEQ carry-over of dioxins and 256 dioxin-like PCBs from feed to eggs. The model could not only predict total sum 257 TEQ, but also the results for sum TEQs of separate groups (dioxins and furans, 258 mono-ortho PCBs and non-ortho PCBs). The model was not tested for individual 259 congeners. The model could successfully be applied for data on the kinetics of total 260 and group sum TEQs of contaminated feed with a much higher level of 261 contamination and a quite different contaminant composition

262 - Absorption of the sum TEQ of the dioxin-like compounds from feed prepared with 263 contaminated oil is quite efficient, being around 90\%; the main elimination route is by yolk fat excretion. Residues in eggs show clearly bi-phasic kinetics, indicating a 
265 fast response after contamination in the order of days followed by a much slower 266 response in the order of months

267 - In contrast, residues in abdominal fat did not show bi-phasic kinetics: the shortlasting fast response phase lacks almost completely. Differences in residue levels in eggs and abdominal fat, expressed as pg TEQ/g fat can be attributed to differences in the kinetics of the corresponding compartments. At steady state, after prolonged exposure to contaminated feed, residue levels are expected to be about the same.

- The model was applied to carry-over of dioxins from two soils incorporated in feed to eggs. The range of absorbed fractions from the two soils was still high but lower than the absorption of dioxins from oil incorporated into feed.

- From model calculations, it is evident that EC MRL values for dioxin-derived TEQ levels in laying hens feed and in eggs are not in accordance/compliance. At least a fourfold reduction is required to guarantee egg levels below the MRL. This should be verified experimentally.

\section{Acknowledgements}

281 This study was sponsored by the Dutch Ministry of Agriculture, Nature Management and 282 Food Quality. 


\section{References}

284 Donoghue DJ, Hairston H, Gaines SA, Bartholomew MJ, Donoghue AM. 1996. Modelling 285 residue uptake by eggs: 1 . Similar drug residue patterns in developing yolks following 286 injection with ampicillin or oxytetracycline. Poultry Science 75; 321-328.

287 Donoghue DJ, Hairston H, Henderson M, McDonald M, Gaines SA, Donoghue AM. 288 1997a. Modelling residue uptake by eggs: Yolks contain ampicillin residues even after 289 drug withdrawal and non-detectability in the plasma. Poultry Science 76; 458-462.

290 Donoghue DJ, Schenk F, Hairston H, Podhorniak LV. 1997b. Modelling residue uptake by 291 eggs: Evidence of a consistent daily pattern of contaminant transfer into developing 292 preovulatory yolks. Journal of Food Protection 60; 1251-1255.

293 Donoghue DJ, Meyers K. 2000. Imaging residue transfer into egg yolks. Journal of the 294 Agricultural and Food Chemistry 48; 6428-6430.

295 Donoghue DJ. 2001. Mechanisms regulating drug and pesticide residue uptake by egg 296 yolks: development of predictive models. Worlds Poultry Science Journal 57; 373-380. 297 Gilbert AB. 1971. The egg: its physical and chemical aspects; In: Bell D.J. and B.M. 298 Freeman, Physiology and biochemistry of the domestic fowl, vol 3; 1379-1399 299 Academic Press, London, New York.

300 Hoogenboom LAP, Kan CA, Zeilmaker MJ, Eijkeren van JCH, Traag WA. Carry-over of 301 dioxins and PCBs from feed and soil to eggs at low contamination levels. Food 302 Additives and Contaminants (submitted).

303 Hoogenboom LAP, Traag WA, Kan CA, Bovee TFH, Weg van der G, Onstenk C, Portier 304 L. 2002. Residues of dioxins and PCBs in eggs following short-term exposure of 305 laying hens to feed from the Belgian crisis. Organohalogen Compounds 57; 241-244.

306 Huygebaert G, Daeseleire E, Grijspeerdt K, VanRenterghem R. 2002. The deposition 307 profile of oxy-carotinoids, fat and PCBs in egg yolks. Arch. Geflügelk. 66 (2002) 216308223.

309 Schuler F, Schmid P, Schlatter Ch. 1997. The transfer of polychlorinated dibenzo-p310 dioxins and dibenzofurans from soil into eggs of foraging chicken. Chemosphere, 34; $311 \quad 711-718$. 
312 Table 1. Optimized parameters for the model for dioxin-like compounds and indicator 313 PCBs. The parameters $q_{c}$ and $q_{f}$ represent the transfer from the central compartment to the 314 fat compartment and vice verse, $y$ and $k$ the elimination via yolk fat and clearance, $F_{a b s}$ the 315 absorbed fraction, and $W_{f}$ the weight of the fat compartment.

\begin{tabular}{lcccccc}
\hline & $q_{c}$ & $q_{f}$ & $y$ & $k$ & $F_{a b s}$ & $W_{f}$ \\
\hline Dioxin and & 0.17 & 0.078 & $0.043<y$ & $0<k<$ & $0.78<$ & $230<W_{f}$ \\
PCB TEQ & & & $<0.055$ & 0.011 & $F_{a b s}<1$ & $<290$ \\
Indicator- & 0.14 & 0.046 & $0.051<y$ & $0<k<$ & $0.68<$ & $220<W_{f}$ \\
PCBs & & & $<0.075$ & 0.022 & $F_{a b s}<1$ & $<320$ \\
\hline
\end{tabular}


319

320

321

322

323

324

325

326

327

328

329

330

331

332

333

334

335

336

337

338

339

340

341

342

343

\section{Legends}

Figure 1. Two compartment PBPK model (A) for the disposition of lipophilic compounds in egg yolk fat of laying hens. $A$ denotes the amounts of contaminant, $V$ the compartments volumes, $P$ partition with respect to blood. The fat compartment serves as a storage for the highly lipophilic contaminants. Fraction $F$ of the dose is absorbed over the gut wall into the central compartment. Elimination is through liver clearance and through excretion with egg yolk fat that is produced with weight $W_{y, f}$ per day with a laying efficiency of $\varepsilon$. These processes can also described by the set of reduced parameters (B) derived from the PBPK model parameters (see Materials and Methods), with $k$ for hepatic clearance, $y$ for excretion through the yolk and $q_{c}$ and $q_{f}$ being the compartment transfer parameters from central to fat compartment and vice versa.

Figure 2. Measured levels (symbols) and computed concentration-time curves based on fitted parameter values for egg yolk (A), and abdominal fat (B). From lower to upper, data and computations correspond to increasing feed contamination levels of $0.34,0.58,0.76$, 1.85 and $3.95 \mathrm{ng}$ of total TEQ (dioxins, furans, non- and mono-ortho PCBs) / $\mathrm{kg}$ feed. Hens were fed contaminated feed during the first 56 days of the experiment.

Figure 3. Levels and calculations for the different groups of contaminants in the yolk fat, based on the data obtained with feed contamination $1.85 \mathrm{ng} \mathrm{TEQ} / \mathrm{kg}$ feed. Upper line (*): total sum TEQ model fit. Next lower line (+): sum TEQ of the group of dioxins and furans; next lower line (x): sum TEQ of the group of non-ortho PCBs; next lower line (o): sum TEQ of the group of mono-ortho PCBs. The last three model calculations were based on the same parameter values that fitted the total TEQ data. At day 55, levels of ten individual 
344 eggs instead of a pooled sample are shown. Hens were fed contaminated feed during the 345 first 56 days of the experiment.

Figure 4. Kinetics of TEQ contamination in eggs (*, upper line) and abdominal fat (+, 349 lower line) during the experimental period of the group fed the diet containing $1.85 \mathrm{ng}$ $350 \mathrm{TEQ} / \mathrm{kg}$ feed (A) and during prolonged feeding with contaminated feed (B). Hens were fed 351 contaminated feed during the first 56 days of the experiment.

354 Figure 5. Contamination levels in egg yolk after administration of ten-fold diluted feed 355 from the Belgian dioxin crisis (Hoogenboom et al. 2002). Hens were fed contaminated 356 feed for 7 days followed by 27 days on clean feed. Model calculations were based on the 357 same parameter values that fitted the total TEQ data. Verification shows the total sum TEQ 358 level of all congener groups (upper line, *), mono-ortho-PCB sum TEQs (next upper line, $359 \mathrm{x}$ ), dioxin sum TEQs (next lower line, o) and non-ortho-PCB sum TEQs (lower line, +).

360 The steps of the staircase show daily contamination level of eggs. 361

362 Figure 6. Levels of indicator PCBs in egg fat (A) and body fat (B) for the 5 different feed 363 levels, and the curve-fit based on the model. Corresponding feed levels from bottom to top 364 were respectively $0.2,2.3,4.3,6.0,14.2$ and $31.7 \mu \mathrm{g} / \mathrm{kg}$ feed. Hens were fed contaminated 365 feed during the first 56 days of the experiment. 


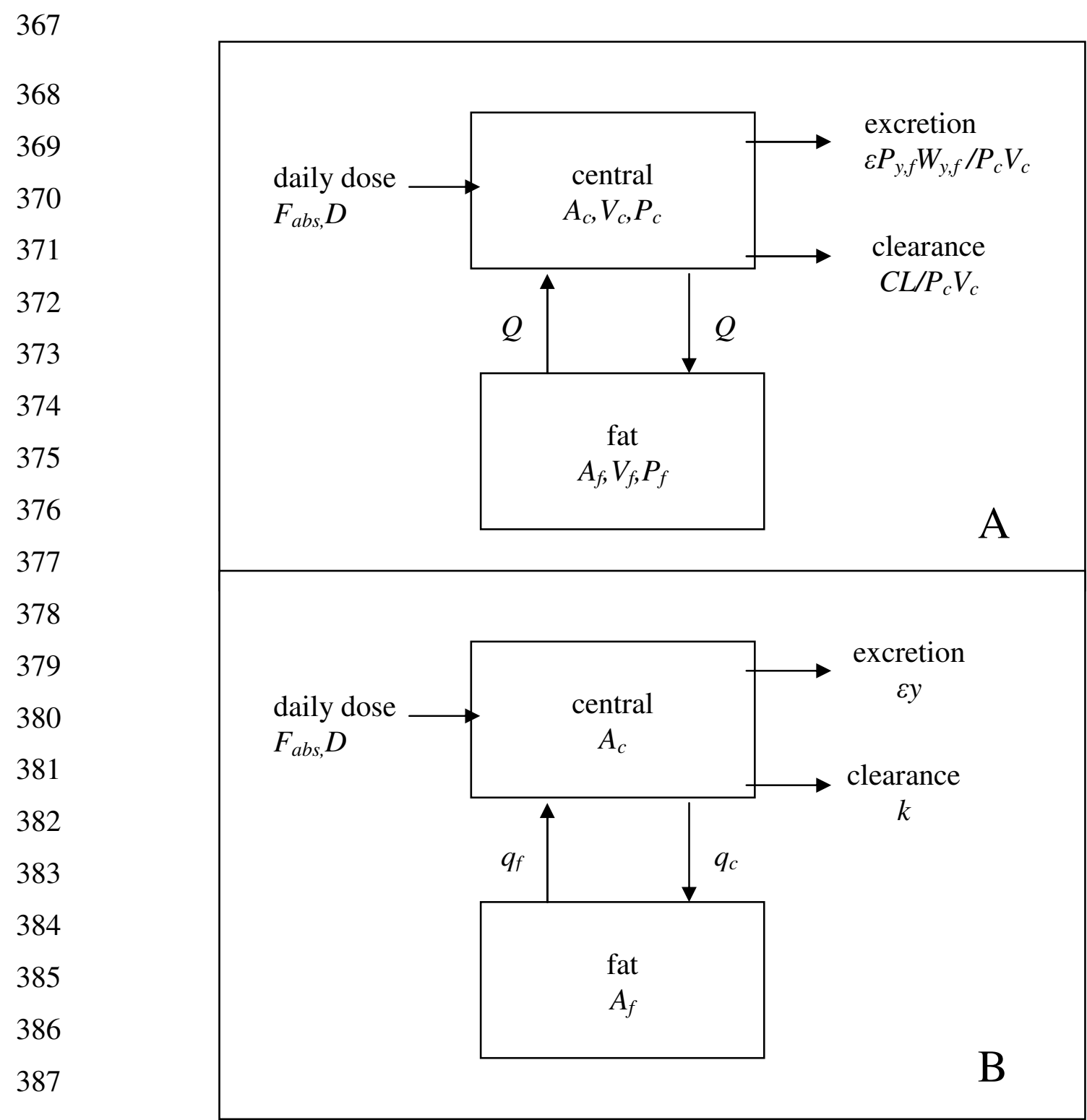

388

Figure 1

390

391

392

393 

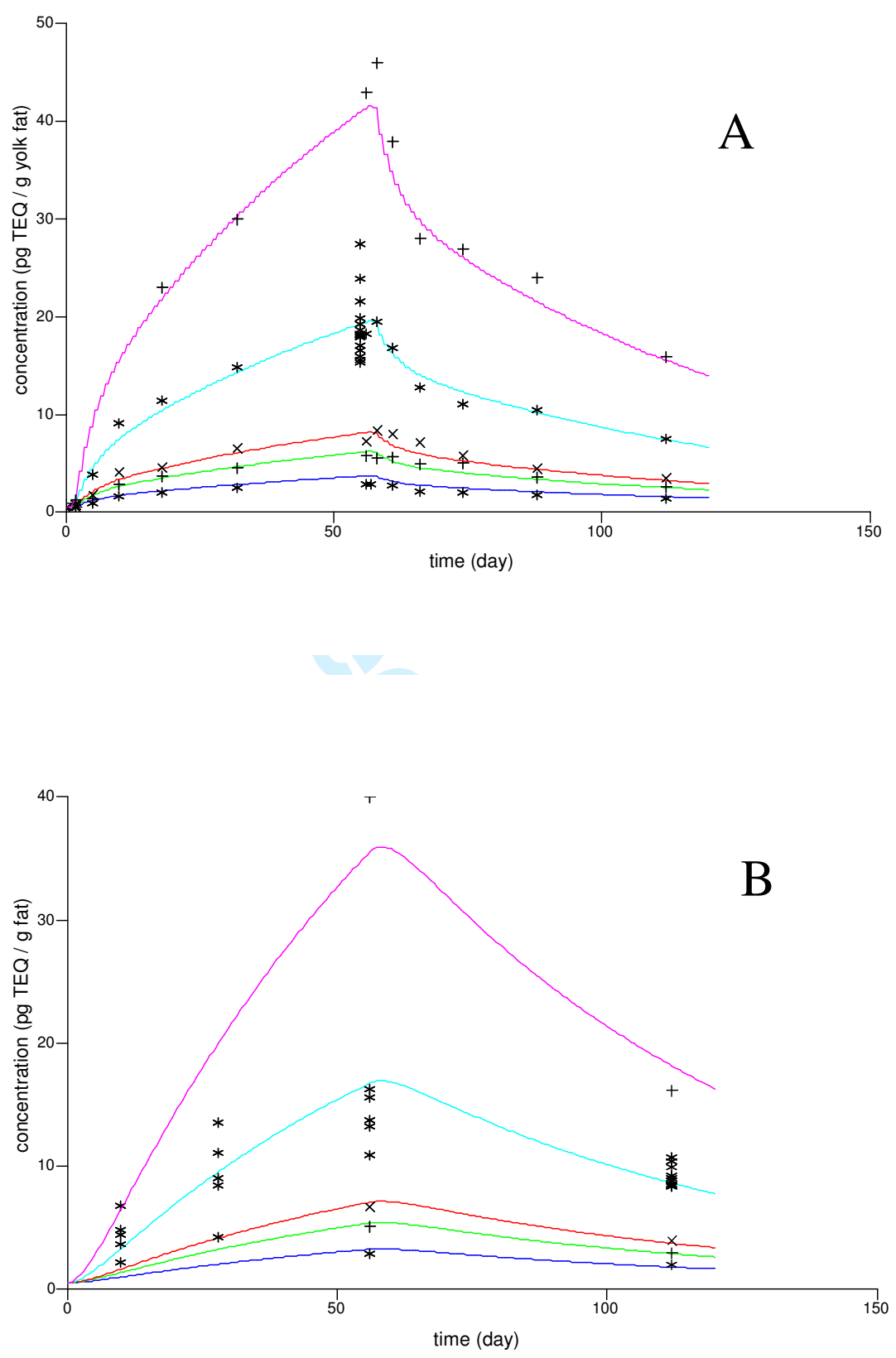

395 Figure 2. 


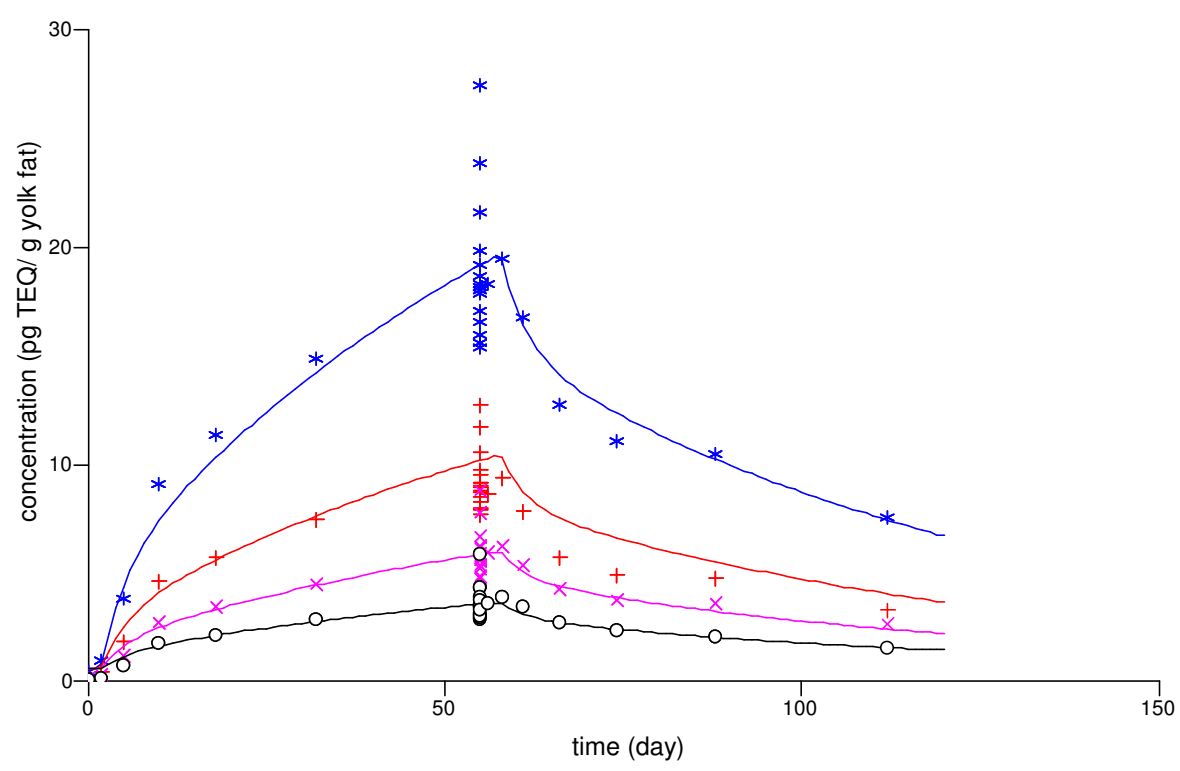

397 Figure 3. 

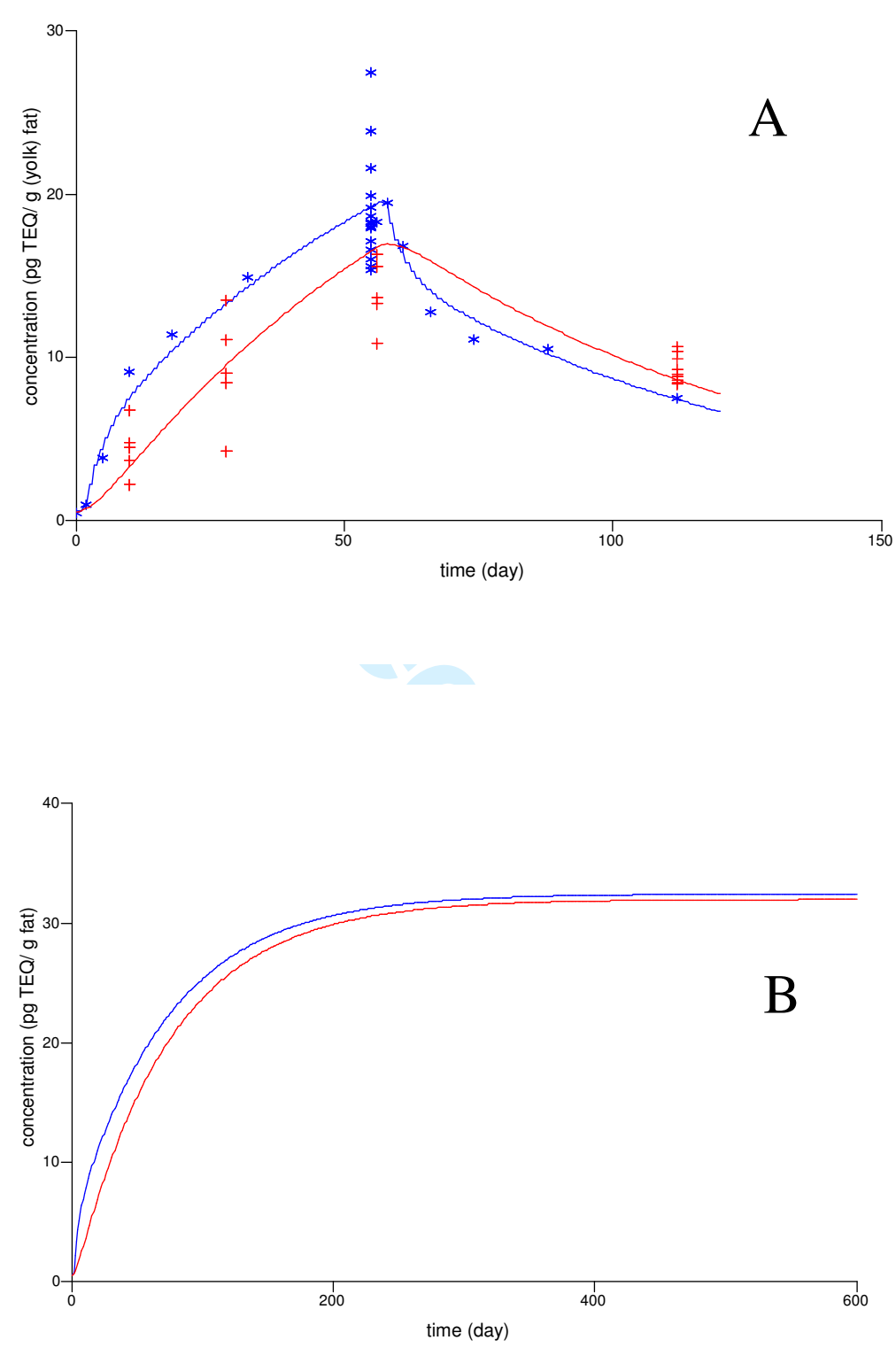

399

$400 \quad$ Figure 4.

401 
402

403

404

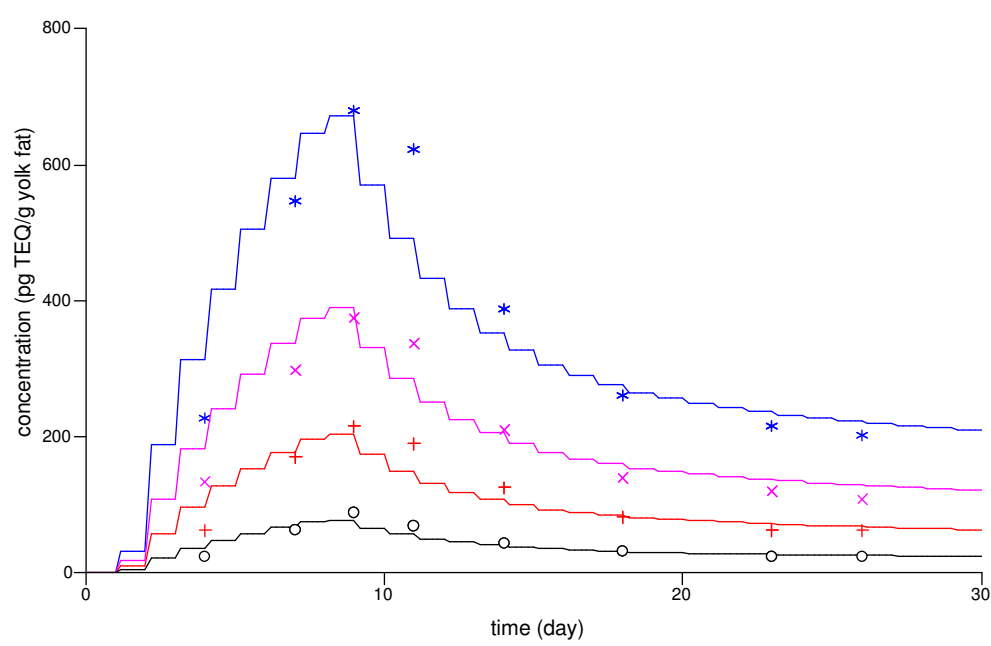

405

406 Figure 5. 

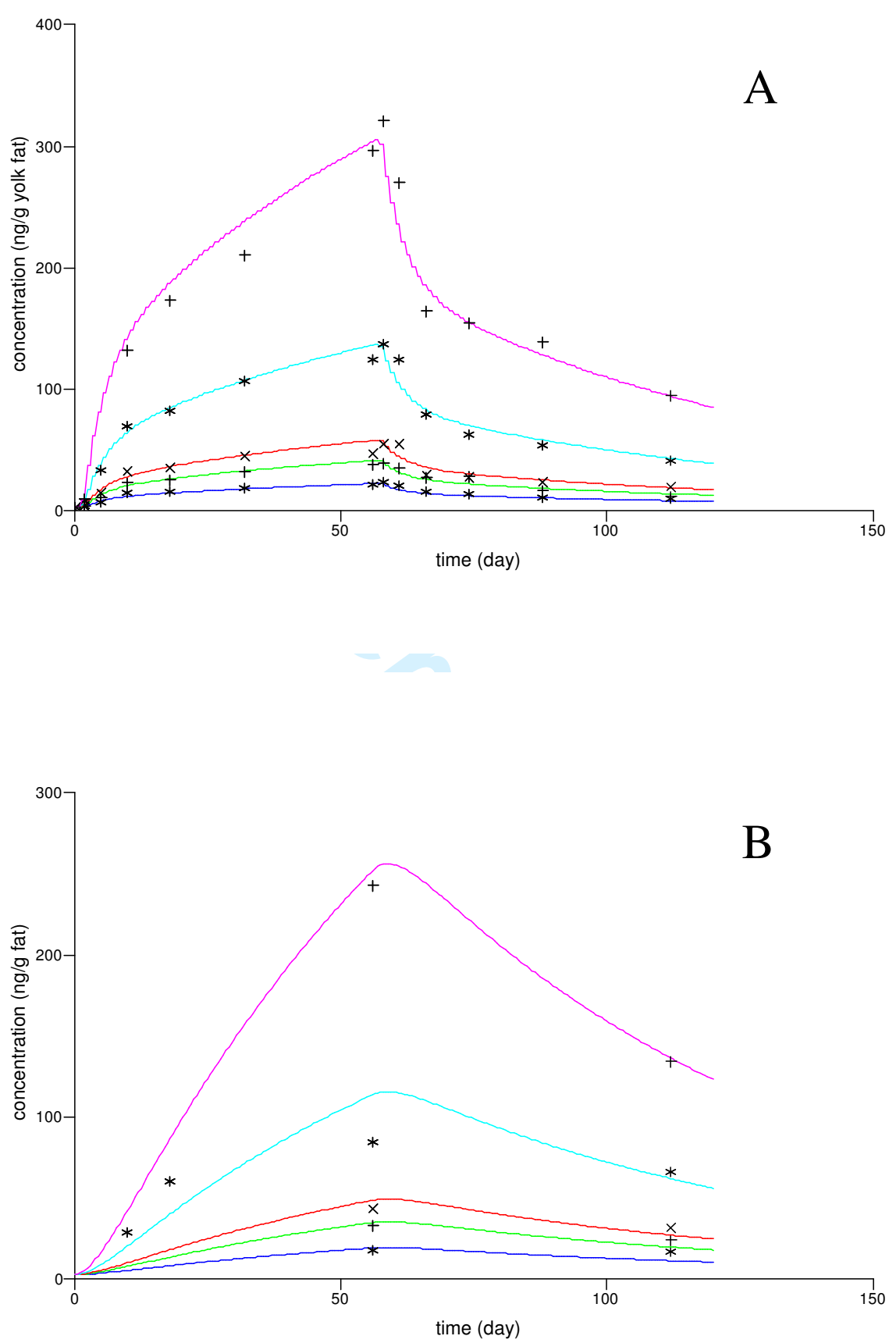

408

409 Figure 6. 